\title{
Direct Identification of Reaction Sites on Ferrihydrite
}

\author{
JEAN-François BoILY ${ }^{1}$ AND XiaOWei SONG ${ }^{1, *}$ \\ 1.Department of Chemistry, Umeå University, Sweden; \\ jean-francois.boily@umu.se \\ *Current address: R\&D, IKEA of Sweden; \\ songxiaowei615@gmail.com
}

Hydroxyl groups are the cornerstone species driving catalytic reactions on mineral nanoparticles of Earth's crust, water, and atmosphere. We here directly identified populations of these groups on ferrihydrite, a key yet misunderstood iron oxyhydroxide nanomineral in natural sciences. This was achieved by resolving an enigmatic set of vibration spectroscopic signatures of reactive hydroxo groups and chemisorbed water molecules embedded in specific chemical environments (Figure 1).

We assist these findings by exploring a vast array of configurations of computer-generated nanoparticles. We find that these groups are mainly disposed along rows at edges of sheets of iron octahedra. Molecular dynamics of nanoparticles as large as $10 \mathrm{~nm}$ showed that the most reactive surface hydroxo groups are predominantly free, yet are hydrogen bond acceptors in an intricate network formed with less reactive groups. Our newly resolved vibration spectroscopic signatures open new possibilities for tracking catalytic reactions on ferrihydrite, directly from the unique viewpoint of its reactive hydroxyl groups.
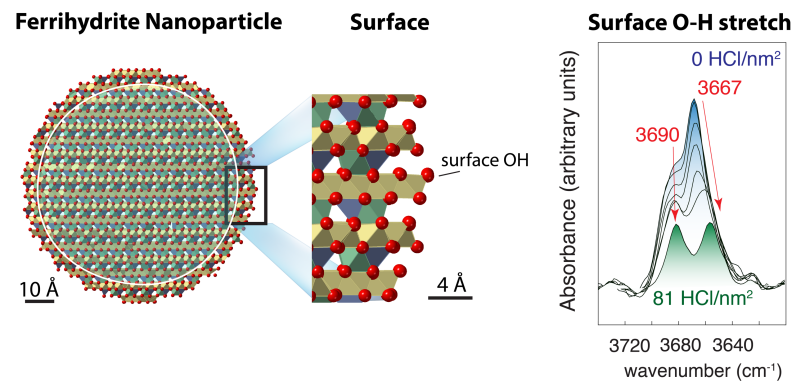

Figure $1 \mathrm{OH}$ groups outcropping ferrihydrite surfaces are predominantly singly- and doubly-coordinated. 